\title{
Striking the balance: Night care versus the facilitation of good sleep
}

\section{Published in British Journal of Nursing, 2012, 21(5): 303-307}

\author{
Authors: Ingrid Eyers*, Emma Young**, Rebekah Luff***, Sara Arber**** \\ * Zentrum Altern und Gesellschaft, University of Vechta, Germany \\ ** Acute Stroke \& Brain Injury Unit, St. Peter's Hospital, Surrey, UK \\ *** School of Social Sciences, University of Southampton, UK \\ **** Centre for Research on Ageing and Gender, University of Surrey, UK
}

\section{Lead Author: Dr. Ingrid Eyers}

Dr. Ingrid Eyers has been involved in the care home sector for 30 years. As a Registered General Nurse she initially worked in intensive care and then transferred to the care of older people in the community. For the last 12 years Ingrid has been undertaking research in care homes. Within the SomnIA (www.somnia.surrey.ac.uk) project her team undertook an in-depth data collection in 10 care homes in order to identify the determinants of poor sleep experienced by care home residents. Ingrid is currently Senior Researcher at the Centre of Research in Ageing and Society at the University of Vechta in Germany.

Contact Details:

Dr. Ingrid Eyers

Zentrum Altern und Gesellschaft

University of Vechta

PO Box 1553

49364 Vechta, Germany

E-mail: ingrid.eyers@uni-vechta.de Tel: + 49444115428 


\begin{abstract}
:
This article presents the key findings from an extensive research project aiming to identify the determinants of poor sleep in care homes. A mixed methods study was conducted in 10 care homes in South East England, which included 2 week daily diaries completed with 145 older residents, observations and interviews with 50 care home staff. The research found that the regular surveillance by qualified nurses and care assistants at night seriously impedes the quality of sleep experienced by older people living in care homes. However, nurses and social care workers have a duty of care and would not be fulfilling their duty if regular checks were not undertaken at night. There is a need for a balance to be struck between enabling older people living in care homes to have a good night's sleep and care home staff adhering to their professional duty of care.
\end{abstract}

Key words: duty of care, sleep, continence care, safeguarding, care homes 


\section{Introduction}

Everyone values a good night's sleep. Professional nurses and social care staff, who have a strong sense of a duty to care, also need to acknowledge and respect that care home residents need a good night's sleep. Based on an extensive study of sleep undertaken in 10 care homes in South East England, involving 145 old people and 50 members of care staff, this article provides an insight into the importance of sleep in old age and considers the conflict between professional accountability and facilitating good sleep for older people living in care homes. The research methodology, which aimed to identify the determinants of poor sleep in care homes, is described and key findings are presented and discussed. The intention is to inform future developments in service delivery in care-giving environments and thereby to improve the quality of life experienced by older people dependent on care and support from professional caregivers.

\section{Older people's perspective}

We all know how difficult the day ahead can be when we have not slept well. Good night time sleep enhances cognitive and physical abilities, reduces the risk of falls, and also enhances quality of life (Livingston et al 1993; Leger 1994; Ersser et al 1999; Busto et al 2001; Martin 2002; Stepanski et al 2003). For older adults these are particularly important aspects of everyday life (Luff et al 2011; Venn and Arber; 2011). However, studies undertaken in both North America and the United Kingdom indicate that the care home environment is not conducive to sleep (Alessi and Schnelle 2000; Kerr et al 2008).

Sleep forms a significant part of the everyday life experienced by older people living in care homes and may dominate their lives. Their experience of sleep in a care home environment can be expected to differ from that of sleep 'at home' in a bed they may have slept in, with their partner for many years, surrounded by furniture, belongings and sounds they know well. Frailty or a medical condition 
is likely to have precipitated their move into residential care, in order for their activities of daily living (ADL) to be supported by care staff. These activities range from waking up in the morning and being prepared for the day, to settling down again at night with the reassurance that help is at hand if needed (Eyers 2007)

\section{Nursing perspective}

One of the main tasks for care home staff is to assist residents in their preparations for the day by supporting them in the vital tasks of getting up, washed and dressed in order to participate in daily life. While providing this support, care staff are also able to enquire how well the resident has slept and monitor their wellbeing, especially in conjunction with their physical and cognitive abilities on that day. In the evening, residents are supported in their preparations for the night by care staff who also facilitate their sleep hygiene. At night, care staff are on hand to safeguard residents, provide continence care and monitor their sleep. In the course of performing all these activities an ethical awareness of a duty to care is to be expected of both qualified nursing staff and care assistants (NMC 2008; Topss England 2004). Whilst all citizens have a duty of care, Registered Nurses (RN) and Social Care Workers are further bound by their professional code of conduct. Neglecting such a core value is seen as professional misconduct (Topss England 2004; NMC 2008). Care giving at night incorporates ensuring that the residents are safe and sleeping soundly. To successfully complete this task, night staff regularly monitor residents’ wellbeing. Night time monitoring is seen to be reassuring to relatives, managers, care staff and regulatory authorities, but it is also important to consider whether it contributes to poor sleep of residents in care homes.

Sleep is such a taken-for-granted yet important aspect of everyone’s life. In recognition of this, the New Dynamics of Ageing Programme (http://www.newdynamics.group.shef.ac.uk/) funded interdisciplinary research into sleep in later life (SomnIA). One of the eight 'Work Packages' (WP) 
within the SomnIA (Sleep in Ageing, www.somnia.surrey.ac.uk) project aimed to identify the factors leading to care home residents' poor sleep at night (WP3). A further WP studied the sleep of community-dwelling older people and the findings enabled comparison between sleep at home and sleep in a care home (Meadows et al 2010; Venn et al 2010). This comparison highlighted the contrast between the two groups of older people and paralleled the findings of Alessi and Schnelle (2000) and Kerr et al. (2008) who documented the poor sleep amongst care home residents. The outcome of research undertaken in WP3 also informed two other 'WPs' within SomnIA that researched potential interventions to improve the sleep of older people living in the community and in care homes. In this article the focus is on the findings from the WP aiming to establish the reasons why older people living in care homes experience poor sleep.

\section{$\underline{\text { Research Methods }}$}

Quantitative and qualitative data were collected from 145 male and female residents aged between 60100 within 10 purposively selected care homes for older people in South East England. In order to ensure a high number of participants able to give informed consent, only care homes registered for $35+$ residents were selected. The ownership of the homes covered nursing and non-nursing homes, local authorities, the voluntary sector, large corporate organisations and small businesses (Table 1). Geographically the homes were situated in suburban and rural areas and aimed to cover a spread of socio-economic groups. All the participating facilities met the required National Minimum Standards for Care Homes for Older People (DH 2003) and consequently were considered to fulfill the statutory criteria set by policy makers at the time of data collection (2008-9). Homes registered to care for 'Elderly Mentally Impaired’ residents were excluded from the study as this would have required a different methodology. Ethical approval for the study was given by the University of Surrey Ethics Committee and Local Authority Social Research Governance. The names of participants used in this paper are pseudonyms to preserve confidentiality. 


\section{Table 1 about here}

Prior to conducting the research, information about the study was provided to the home management, all care home staff, residents and their relatives. On the day that the data collection commenced, a presentation was made to residents and staff in each home. Residents who had volunteered were cognitively able to participate in the study and each had a detailed conversation with the researchers before signing the consent form to participate in the study. Participation involved wearing an actiwatch (small activity and light exposure monitors) to record levels of movement continuously for 14 days and keeping a daily diary over a period of two weeks denoting their everyday activities. As the majority of the 145 participants were not able to personally maintain the diaries, researchers visited daily and chatted with the residents to elicit information about activities during the previous 24 hours. The diaries were maintained in a standardised questionnaire format which facilitated analysis using SPSS. The questions asked daily included how well the participant had slept, how often they had got up during the night and if they were disturbed during the night. Further information was collected to establish what time the resident went to bed and got up, what activities they had undertaken during the day and what they had eaten. Collecting data in this way also facilitated keeping detailed field notes during the 2 week period of data collection.

An observational study was also conducted, covering dawn, dusk and during the night time in each of the participating care homes, totalling 250 hours of observations. Analysis of the observational data and field notes enabled the researchers to evaluate the validity of the daily information provided in the diaries. 
Each diary also recorded demographic information and the participants' dependency level based on the amount of time care staff stated was required to provide care each day (Eyers 2000; 2007). As not all care staff were qualified nurses this was the most reliable, tested form of data collection. Information about individual participants' continence care plans was also elicited. Audio tape recorded semi-structured interviews were conducted with all 10 care home Managers and a further 50 members of day and night care staff. The structure of the interviews contributed towards identifying organisational factors influencing sleep and the perception that care staff had about residents' sleep. The interviews were fully transcribed and analysed using NVivo 8. To gain a deeper understanding of the work load night staff encountered, additional data was collected from the Managers to establish the care needs of all the residents in each home.

\section{Figure 1 about here}

This multi-method approach facilitated ‘rigor without rigidity’ (Fielding 2009: 428) for this study with vulnerable older people living in a complex community. Bringing together the qualitative and quantitative data facilitated data analysis that provided a deeper understanding of care home life and

the interaction between older people and the staff employed to support them (Figure 1). The findings aim to inform policy and practice development as well as the other WPs within the SomnIA project. To integrate a practitioners' perspective into our analysis, two well attended 'Day Workshops' were held, which enabled discussion about the findings with care home managers and night staff.

\section{Quantitative Data Findings}

Diary data was collected from 145 participants who completed all or part of the two week daily diaries. There were 105 women and 40 men, covering an age range of 60 to 100 with the strongest 
representation of both genders in the age group 85-89 (Figure 2).

\section{Figure 2 about here}

Information about the participant's health care needs was provided by care staff and indicated that 27 needed under 90 minutes physical assistance per day whilst 79 received 90-180 minutes of physical care and support per day. Three to five hours of 'hands-on' care was provided to 30 residents, and 6 participants received over 5 hours of daily assistance to meet their health care needs. Data relating to dependency was missing from 3 participants.

Background data collected about the care homes provided by care home matron/managers indicated that assisting and supporting residents to use the toilet was, alongside assistance in getting washed and dressed, one of the main forms of support provided to residents by care staff. From the observational data, it was clear that assistance with toileting and continence care is provided day and night. The data strongly indicated that such a sensitive, very personal bodily function that a healthy independent person undertakes in privacy, is a core element in the everyday (and night) life of vulnerable old people living in care homes.

A key finding from the diary data was that the mean length of time care home residents spent in bed at night was 10 hours 50 minutes. However it would be erroneous to equate the hours in bed with long hours slept, as the majority of the participating residents reported spending a number of hours awake in bed each night (Luff et al 2011). 


\section{Figure 3 about here}

The actigraphy (data from actiwatches) analysis established that many participating residents experienced fragmented sleep. Figure 3 shows an example of the actigraphic record for 48 hours of a typical care home resident with fragmented sleep. The spikes represent movement, and therefore when the resident is awake. Only where there is a flat line on the horizontal axis is the resident likely to be asleep. Figure 3 indicates that this residents' pattern of movement is little different during the day and the night-time on the Saturday, in particular there are peaks of movement at two hourly intervals during the night, probably reflecting routine checks by care staff. On the Sunday, there appear to be periods of sleep from $2.00 \mathrm{am}$ to $5.30 \mathrm{am}$, and also in the afternoon from $1.30 \mathrm{pm}$ to 4.00pm. The observational data and interviews provide an insight into potential reasons behind residents’ disturbed sleep. However, an indication comes from the diary data which showed that $65 \%$ of sleep disturbances were reported by residents to be related to toilet/continence care. This factor links with the qualitative findings; both the interviews and observations indicated that continence care was a dominant aspect of care home life.

\section{$\underline{\text { Qualitative Data Findings }}$}

Based on grounded theory principles (Strauss and Corbin 1998), a key theme identified within the qualitative observational and interview data was the frequency of night-time monitoring by staff, which was driven by two requirements:

Dead or alive? Reliably monitored by entering the bedroom to visually assess residents’ wellbeing (are they still breathing?)

Wet or dry? Reliably monitored through the physical evaluation of the continence pad or sheet. 
Not heeding these aspects of care giving could be interpreted to mean care staff are failing in their duty of care. Regular hourly checks were expected by managers and routinely performed by care staff, which was evident from the interview, observational and actigraphy data.

Based on the interview data collected and discussions during the Day Workshops attended by Care Home Managers, it was clear that night staff generally do not intentionally wake residents at night. Interviewed care staff also tended to believe that 'their residents' sleep well. They made this evaluation on the basis of the following three key points:

- 'their residents' did not take 'sleeping tablets'

- 'their residents' did not use the buzzer during the night

- the sound of 'their residents'” snoring

A care home manager said:

'They [residents] are checked hourly [during the night]... unless residents specifically request that we do not go into the room... they are checked hourly...'.

Madge, a care assistant said:

'... Checking on them... that's our work, we have to look after them'.

However, to counterbalance the above two interview extracts thought needs to be given to the following statement made by a resident, Stanley:

'... When the staff come into my room, I wake up'. 
Considering that a high proportion of the participants experienced fragmented sleep, it becomes clear that there is a potential area of conflict between care staff facilitating a good night's sleep and fulfilling a duty of care. Regular surveillance of night time sleep by care staff restricts residents' experience of restorative sleep, which could enhance their cognitive and physical abilities during the day.

\section{$\underline{\text { Conclusion }}$}

This comparatively large scale study of care home life in England and the mixed method research approach aimed to ensure the collection of ethical, reliable and valid data in a complex environment involving vulnerable people. This study set out to identify determinants of poor sleep experienced by older people living in care homes. The findings contribute to understanding the reasons for poor sleep identified in other studies (Alessi and Schnelle 2000; Kerr et al 2008).

On the basis of the evidence from this research it is important to address the issue of how care at night is provided so that it does not disrupt residents' sleep, while night staff still fulfill their duty of care. However, a limitation of this study is the lack of detailed enquiry into the actual understandings that care home staff have of a 'duty of care'. There is a need to consider what can be done to ensure the development of best practice that enables care home residents to sleep well while also reassuring care home staff, managers and relatives that, at night, all residents are safely asleep in dry beds. It is equally important to value the positive impact of good sleep on a resident's physical and cognitive abilities during the day. A good night's sleep for residents could improve both residents' and care staffs' day time experiences. Facilitating a good nights sleep furthermore exemplifies the effective implementation of person-centred care (Innes et al 2006).

Further research is recommended to support and encourage the use of readily available night time 
sensors and to comprehensively review routine daytime and night-time care giving procedures to support the implementation of person centred care that enables restorative sleep. A key research goal should be to develop procedures by which care staff can fulfill their 'duty of care' for the well-being of residents at night, but without disturbing resident's sleep. Further research is called for to gain a deeper understanding of the links between policy and practice relevant to professional Codes of Practice established to provide night-time guidance within a wide spectrum of health and social care settings.

Policy makers, care service regulators, managers, nurses and care assistants jointly have a duty of care to address this vital aspect of care home life. To succeed, this would require the regulators' support, and for the importance of sleep to be explicitly included in care staff training programs. It could well be a hard day’s night to ensure care home residents can sleep like a log. 


\section{References}

Alessi C A, Schnelle J (2000) Approach to sleep disorders in the nursing home setting Sleep Medicine Reviews 4(1) 45-56

Busto U, Spoule B, Knight K, Hermann N (2001) Use of Prescription and Nonprescription Hypnotics in a Canadian Elderly Population. Canadian Journal of Clinical Pharmacology, 8 (4), 213-221

Department of Health (2003) Care Homes for Older People, National Minimum Standards, London, HMSO,

Ersser S, Wiles A, Taylor H, Wade S, Walsh R, Bently T. (1999) The sleep of older people in hospital and nursing homes. Journal of Clinical Nursing, 8: 360-368,

Eyers I (2000) 'Education and Training. Do they really, really want it? A comparative study of care home staff in England and Germany', Education and Ageing, 15(2): 159-175

Eyers I (2007) 'Extracting the Essence of Formal Caregiving: A Comparative Study of Formal Care Givers in English and German Care Homes’ in Paoletti, I. (ed) 'Family caregiving to older disabled people: relational and institutional issues’ Novascience, New York, USA

Fielding N (2009) 'Going out on a limb: Postmodernism and Multiple Method Research' in Current Sociology 57(3):427-447.

Innes A, Macpherson S, McCabe L. (2006) Promoting Person-centred care at the front line, York, Joseph Rowntree Foundation

Kerr D, Wilkinson H, Cunningham C. (2008) Supporting older people in care homes at night. Joseph Rowntree Foundation, York.

Leger D (1994) The Cost of Sleep-Related Accidents: A Report for the National Commission on Sleep Disorders Research, Sleep, 17: 84-93

Livingston G, Blizard B, Mann A (1993) Does sleep disturbance predict depression in elderly people - a study in inner London. British Journal of General Practice, 43: 445-448

Luff R, Ellmers T, EyersI, Young E, Arber S (2011) 'Time spent in bed at night by care homes 
residents.' Ageing and Society 31(7): 1229-1250

Martin P (2002) Counting Sheep Harper Collins, London

Meadows R, Luff R, Eyers I, Venn S, Cope E. and Arber, S. (2010) An actigraphic study comparing community dwelling poor sleepers with non-demented care home residents Chronobiology International 27 (4): 842-854

NMC (2008) Duty of Care, http://www.nmc-uk.org/Nurses-and-midwives/Advice-bytopic/A/Advice/Duty-of-care/ (Accessed 01.06.11)

Toppss England (2004) The National Occupational Standards for Social Work http://www.skillsforcare.org.uk/nmsruntime/saveasdialog.aspx?IID=774\&sID=161 (accessed 1.12.11)

Stepanski E, Rybarczyk B, Lopez M, Stevens S (2003) Assessment and treatment of sleep disorders in older adults: A review for rehabilitation psychologists Rehabilitation Psychology 48: 23-36

Strauss, A. and Corbin, J. (1998) Basics of Qualitative Research Techniques and Procedures for Developing Grounded Theory Sage Publications, London

Venn S, Arber S (2011) 'Daytime sleep and active ageing in later life’ Ageing and Society, 31(2): 197-216.

Venn S, Luff R, Ellmers T, Arber S, Eyers I (2010) ‘Optimising quality sleep among older people in the community and care homes: Some key findings’ Generations Review, 20: 4. $<$ http://www.britishgerontology.org/10newsletter4/research5.asp > 
Table 1: Characteristics of $\mathbf{1 0}$ studied care homes

\begin{tabular}{|l|l|l|l|l|l|l|l|l|l|l|}
\hline Selection Criteria & $\mathbf{1}$ & $\mathbf{2}$ & $\mathbf{3}$ & $\mathbf{4}$ & $\mathbf{5}$ & $\mathbf{6}$ & $\mathbf{7}$ & $\mathbf{8}$ & $\mathbf{9}$ & $\mathbf{1 0}$ \\
\hline No. of beds & $\mathbf{6 0}$ & $\mathbf{4 4}$ & $\mathbf{3 8}$ & $\mathbf{3 8}$ & $\mathbf{7 2}$ & $\mathbf{4 3}$ & $\mathbf{5 8}$ & $\mathbf{7 4}$ & $\mathbf{8 2}$ & $\mathbf{4 7}$ \\
\hline $\begin{array}{l}\text { Vol. Sector } \\
\text { Owner }\end{array}$ & $\checkmark$ & & & & & & & & & \\
\hline $\begin{array}{l}\text { Local Authority } \\
\text { Owner }\end{array}$ & & $\checkmark *$ & $\checkmark *$ & $\checkmark *$ & & & & & & \\
\hline $\begin{array}{l}\text { Corporate } \\
\text { Owner }\end{array}$ & & & & & $\checkmark * *$ & $\checkmark * *$ & $\checkmark * *$ & $\checkmark$ & $\checkmark$ & \\
\hline Small Business & & & & & & & & & & $\checkmark$ \\
\hline Purpose build & $\checkmark$ & $\checkmark$ & $\checkmark$ & $\checkmark$ & $\checkmark$ & $\checkmark$ & $\checkmark$ & $\checkmark$ & $\checkmark$ & $\checkmark$ \\
\hline Conversion & & & & & & & & $\checkmark$ & $\checkmark$ & $\checkmark$ \\
\hline
\end{tabular}

*same ownership ** same ownership 
Figure 1: Methodology and Conceptual Framework

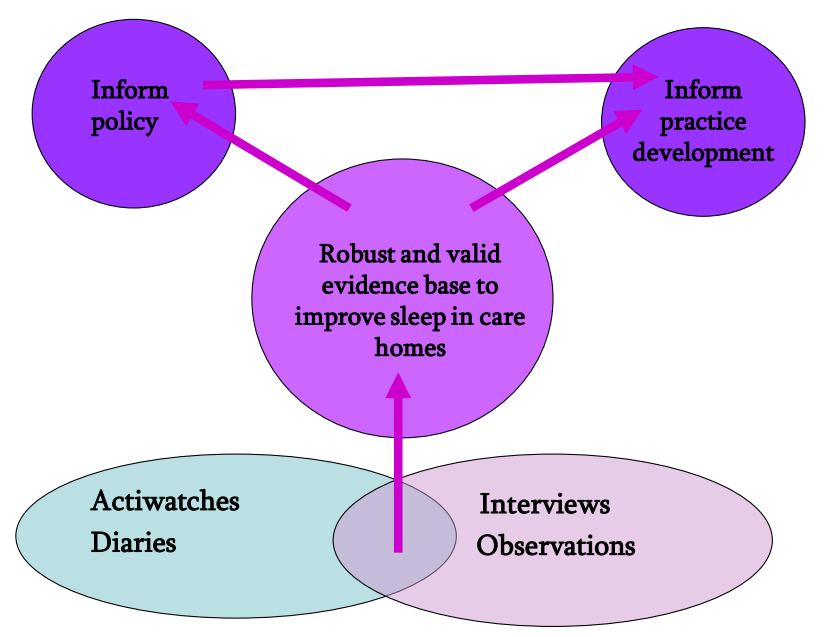


Figure 2. Age and Gender of Care Home Residents in sample

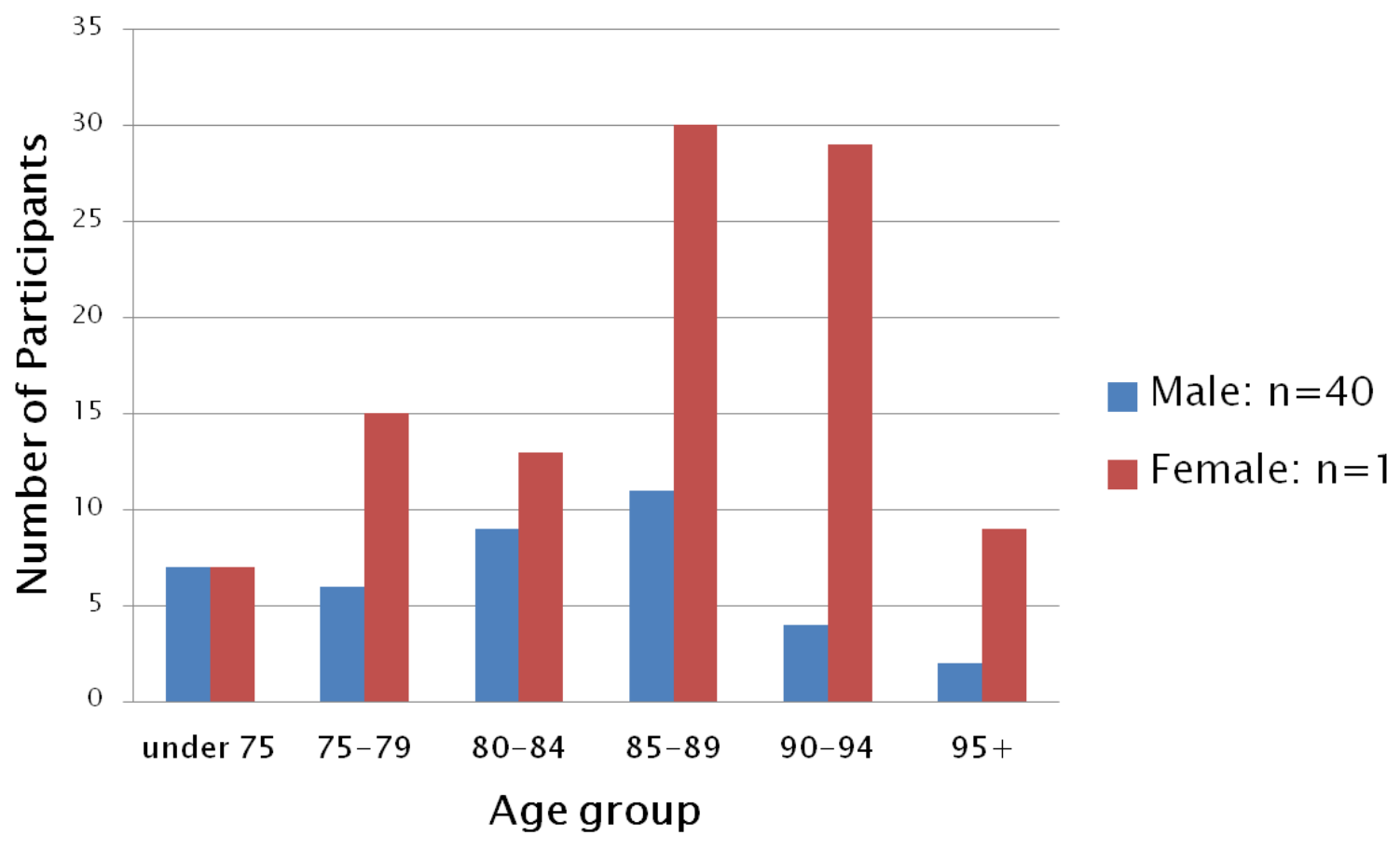


Figure 3. Actigraph Exemplifying a care home resident with Fragmented Sleep (for two periods of 24 hours)

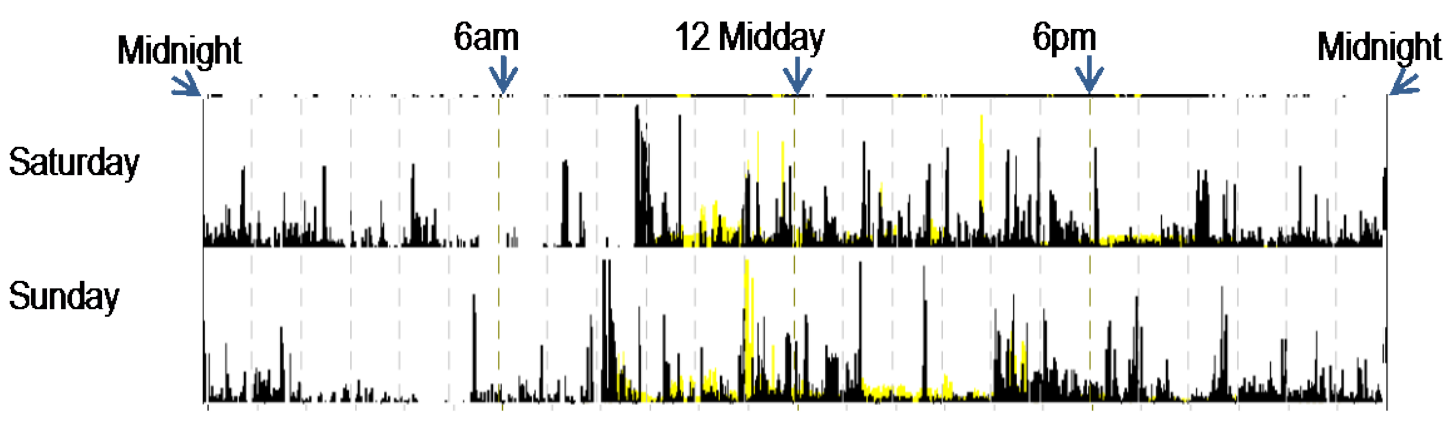

